\title{
Subtraction method for intravital two-photon microscopy: intraparenchymal imaging and quantification of extravasation in mouse brain cortex
}

\author{
Pascale Vérant \\ CNRS UMR 5588 \\ Laboratoire Spectro \\ 38402 Saint Martin d'Hères, France \\ and \\ Campus Universitaire de Saint-Jérome \\ Institut Fresnel \\ Marseille, France
}

\section{Raphaël Serduc \\ Boudewijn van der Sanden \\ Rémy Chantal \\ Clément Ricard \\ Jonathan A. Coles \\ INSERM, UMR-S 836 \\ Grenoble Institut des Neurosciences \\ Grenoble 38043, France and \\ Université Joseph Fourier \\ Grenoble, France}

\author{
Jean-Claude Vial \\ CNRS UMR 5588 \\ Laboratoire Spectro \\ 38402 Saint Martin d'Hères, France \\ and \\ Université Joseph Fourier \\ Grenoble, France
}

\begin{abstract}
Brain pathologies, including stroke and tumors, are associated with a variable degree of breakdown of the blood-brain barrier (BBB), which can usefully be studied in animal models. We describe a new optical technique for quantifying extravasation in the cortex of the living mouse and for imaging intraparenchymal tissue. Leakiness of the BBB was induced by microbeam x-irradiation. Two fluorescent dyes were simultaneously infused intravenously, one of high molecular weight (fluorescein-labeled dextran, $70 \mathrm{kDa}$, green fluorescence) and one of low molecular weight (sulforhodamine B, 559 Da, red fluorescence). A two-photon microscope, directed through a cranial window, obtained separate images of the two dyes in the cortex. The gains of the two channels were adjusted so that the signals coming from within the vessels were equal. Subtraction of the image of the fluorescein-dextran from that of the sulforhodamine B gave images in which the vasculature was invisible and the sulforhodamine $B$ in the parenchyma could be imaged with high resolution. Algorithms are presented for rapidly quantifying the extravasation without the need for shape recognition and for calculating the permeability of the BBB. Sulforhodamine B labeled certain intraparenchymal cells; these cells, and other details, were best observed using the subtraction method. (C) 2008 Society of Photo-Optical Instrumentation Engineers. [DOI: 10.1117/1.2870083]

Keywords: biomedical optics; microscopy; image processing; fluorescence.

Paper 07189SSR received May 25, 2007; revised manuscript received Aug. 3, 2007 ; accepted for publication Aug. 30, 2007; published online Feb. 28, 2008.
\end{abstract}

\section{Introduction}

The endothelium of brain capillaries regulates the exchange of molecules between the blood and the parenchyma. The passage of most molecules is hindered, although specific transport mechanisms exist for certain molecules, notably glucose. The blood brain barrier (BBB) is of great importance clinically for two main reasons. First, many pathologies are associated with an increase in the permeability to large molecules. This leads to their entry, together with water, into the parenchyma, leading to vasogenic edema and an increase in intracranial pressure that has harmful, even fatal, consequences. Vasogenic edema is a major complication in brain pathologies including trauma, stroke, tumors, subarachnoid hemorrhage, and neuroinflammation. ${ }^{1}$

A second reason for clinical interest in the BBB is that it not only restricts access of systemically delivered drugs to the nerve cells, but also the capillary endothelium possesses transporters of the ABC family, called multi-drug-resistance transporters, that remove, via the cytoplasm of the endothelial cells, those drug molecules that do reach the parenchyma. ${ }^{2}$ The dominant transporter in the brain is the P-glycoprotein, or

Address all correspondence to: Jean-Claude Vial, CNRS UMR 5588 Laboratoire Spectro 38402 Saint Martin d'Hères, France; Tel: 0476635866; Fax: 0476635495 Email: jean-claude.vial@ujf-grenoble.fr
Mdr1. Manipulation of the BBB so as to increase the entry of drugs and to reduce their evacuation is a major research goal for the treatment of several pathologies, including epilepsy and migraine. $^{3-5}$

The capillary endothelium is known to respond to signals from surrounding cells, notably the astrocytes, and it is therefore important to measure transport across the BBB in vivo. ${ }^{3,6}$ Post mortem imaging on brain sections of the distribution of labeled molecules (such as ${ }^{14} \mathrm{C}$ sucrose) is still a common technique, but it gives a result for only one time-point per animal. $^{7-9}$ Magnetic resonance imaging (MRI) can follow extravasation of contrast agents, such as gadolinium complexes, that modify the magnetic susceptibility. ${ }^{9,10}$ However, only a few contrast agents are available, and in general, they are very different from biological molecules. Positron emission tomography $(\mathrm{PET})^{11}$ allows a large range of molecules to be studied, but they must be labeled with positron-emitting isotopes. Also, these two techniques have poor spatial and temporal resolution.

In vivo optical imaging of extravasation of fluorescent marker molecules offers promise of much better resolution in space $(<1 \mu \mathrm{m})$ and in time (msec). Many different fluorescent molecules are available, and fluorophores can be attached

1083-3668/2008/13(1)/011002/11/\$25.00 @ 2008 SPIE 
to other molecules of biological interest. With conventional light microscopy, it has been possible to image only vessels no deeper than tens of microns from the surface, as in skinfold tumor models ${ }^{12}$ or pial vessels on the brain surface, ${ }^{5,13}$ and also vessels in the retina. ${ }^{14}$ However, the properties of these vessels differ from those of vessels within the brain itself. ${ }^{3}$ The advent of multiphoton microscopy, which uses excitation at near-infrared wavelengths, ${ }^{15}$ makes possible in vivo imaging with submicron resolution at depths up to several hundred microns below the brain surface ${ }^{16}$ and has allowed detection of localized extravasation of fluorescent dye in the mouse cortex in vivo. ${ }^{17}$ However, one problem is that unless there is complete breakdown of the BBB, the concentration of dye within the vessels is vastly higher than in the extravascular tissue. In order to detect the extravascular dye, it is necessary to use a sensitivity so high that the fluorescence signal from the vessels themselves tends to contaminate, or even swamp, the true extravascular signal.

We show here that this problem can be avoided by simultaneously infusing two fluorescent dyes, one with properties such that it does not cross the leaky BBB (e.g., a high molecular weight) and one that does. The two dyes both fluoresce when excited with intense near-infrared light, but they are chosen to emit at different wavelengths so that the distributions of the two can be imaged separately. We increased the permeability of the $\mathrm{BBB}$ of mice by irradiating them with $\mathrm{X}$-ray microbeams from a synchrotron. ${ }^{18}$ We show that by balancing the gains of the two image channels and subtracting the image of the nonextravasating dye from that of the extravasating dye, the distribution of extravascular dye can be imaged accurately. We derive a formula, which turns out to be very simple, for calculating the $\mathrm{BBB}$ permeability from the difference signal. The method could in principle be used with conventional epifluorescence microscopy of vessels close to the surface of the brain, but it offers more potential when two-photon microscopy is used to image deep into the cortex.

In the course of this work, we found by serendipity that our extravasating dye, sulphorhodamine B, also strongly labeled objects in the tissue. These too were best seen using the subtraction method. This observation raises the possibility that labeling of specific brain structures (for example, astrocytes) might be done in vivo via a vascular route. The identification of the objects labeled by sulforhodamine B, and the conditions for labeling them, will be described elsewhere. ${ }^{19}$

\section{Outline of the Theory}

In this section, we outline the principle of the method and give the expressions necessary to calculate the specific permeability $P$ of the BBB. A complete derivation is given in Sec. 7 .

\subsection{Qualitative Subtraction Imaging}

A fluorescent marker dye is infused intravenously. A small fraction crosses the BBB and appears at a relatively low concentration in the extravascular space. We want to image this low concentration in the presence of a much higher concentration in a dense network of capillaries. Although two-photon microscopy offers excellent spatial resolution by conventional criteria (such as the Rayleigh criterion for the separation of two points), these criteria are not relevant to the present case in which the image of a fluorescent capillary extends, with a
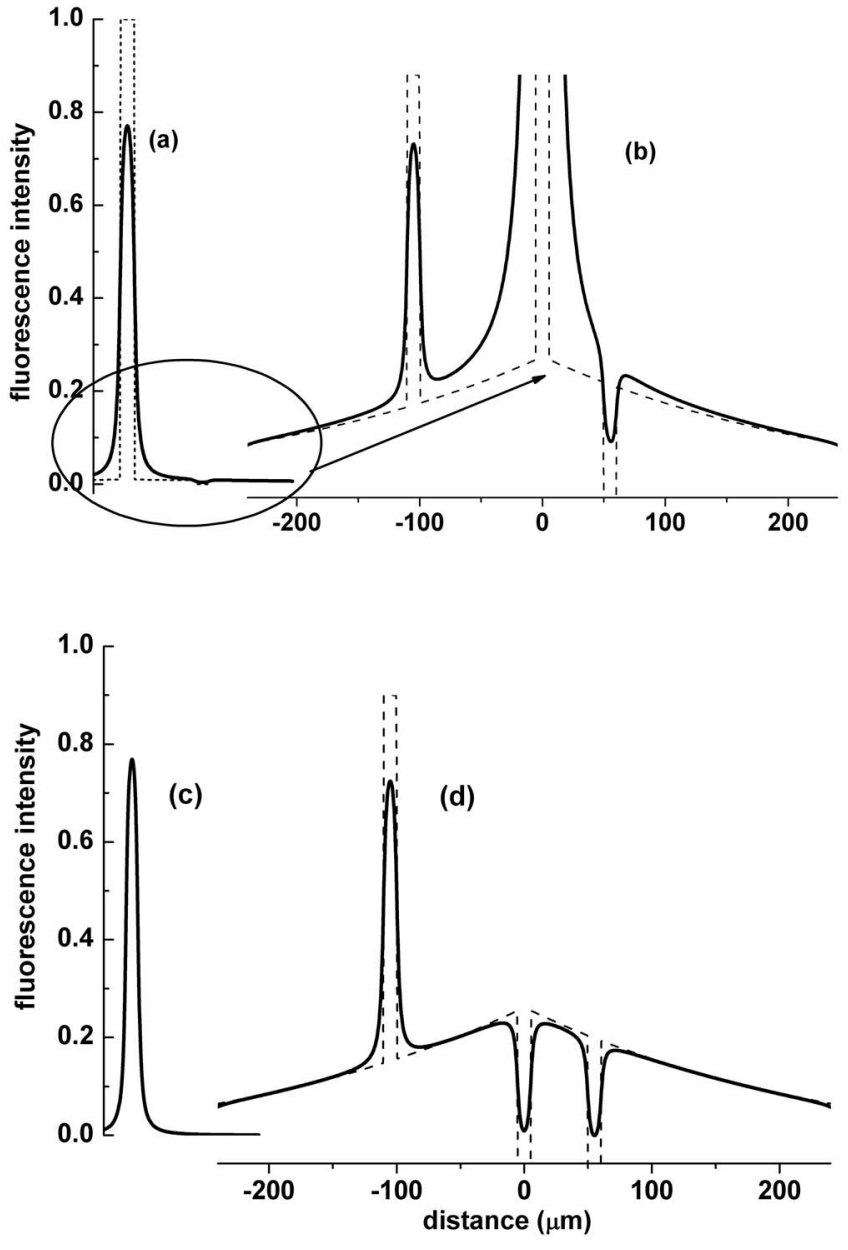

Fig. 1 Schematic numerical simulation of the differential imaging method (a) represents a section of a capillary in the presence of diffusible dye (dashed line) and its convolution with the laser point spread function (PSF, solid line). A small amount of dye has extravasated. The PSF was for the experimental conditions used: two-photon absorption of 800-nm light with a numerical aperture of 0.95 . (b) shows part of (a) on an expanded scale showing the dye extravasation and small hyperstained and unstained regions (dashed line) and their image (solid line). In this figure, it is difficult to separate the extravasation and PSF-induced broadening. (c) is equivalent to (a), but for a dye that remains within the capillary. ( $d$, solid line) is the numerical subtraction (a) to (c), while the dashed line is the distribution of the extravasating dye.

low intensity, well beyond the geometrical limits of the capillary [solid lines in Figs. 1(a) and 1(b)]. Hence, a significant part of the acquired signal attributed to a point in the extravascular space comes, in practice, not from the extravascular dye, but from the intense fluorescence of the much more concentrated dye within the capillaries in the object plane [difference between solid and dashed lines in Fig. 1(b)] or, in threedimensional (3-D) imaging, from blood vessels above or below the object plane. We overcome this problem by the use of a second dye that remains intravascular (NP, for nonpermeant). The mouse is injected with a mixture of the two dyes, in our case, SRB (P, for permeant) and FITC-dextran (NP). A region of the cortex is imaged using two-photon microscopy to give separate images of P and of NP. The essence of the method is to adjust the gains of the two detection chains so 

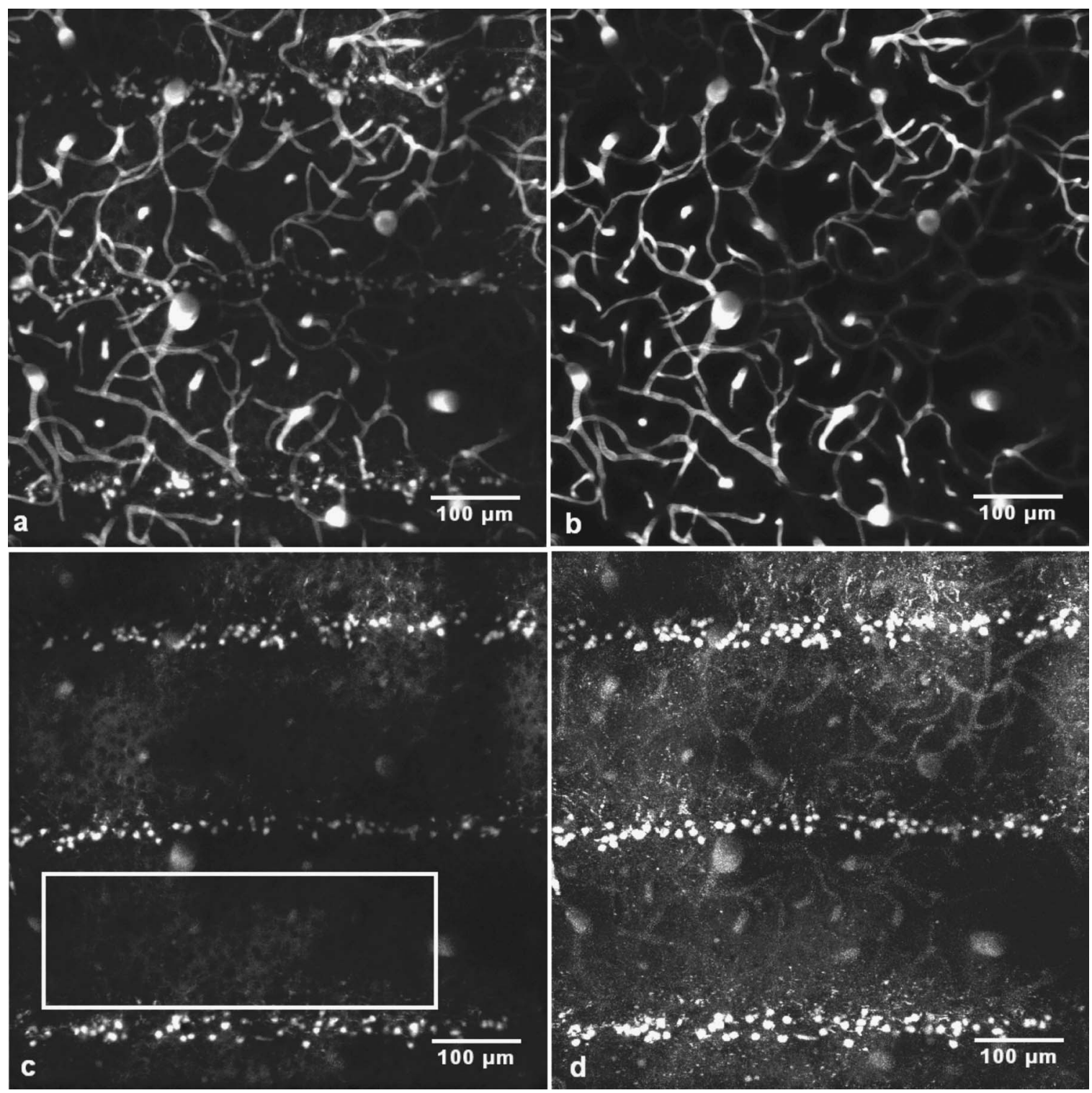

Fig. 2 (a) and (b) z-projection (maximum intensity) of stacks of images acquired in vivo from 150 to $300 \mu \mathrm{m}$ below the dura (in steps of $2 \mu \mathrm{m}$ ) in the left parietal cortex of a nude mouse $48 \mathrm{~h}$ after microbeam irradiation. The mouse was injected with $100 \mu \mathrm{l} \mathrm{of} 100 \mathrm{mg} \mathrm{ml}^{-1} \mathrm{FITC}-\mathrm{dextran}$ solution and $50 \mu \mathrm{l}$ of a $5 \mathrm{mg} \mathrm{ml}^{-1}$ SRB solution. The observation was made $10 \mathrm{~min}$ after the dye injection. (a) is obtained from the emission of the sulforhodamine B dye. (b) is obtained from the emission of the FITC-dextran dye. (c) is the z-projection (average intensity) of the difference stack. (d) is the z-projection (maximum intensity) of the difference stack. In images (c) and (d), the gain has been increased until the brightest pixels are just saturated.

that the two signals from the intravascular space are equal. When the NP signal in each pixel is subtracted from the P signal, the remaining signal in the extravascular space is almost purely from the extravascular P molecules [Fig. 1(d), Figs. 2(c) and 2(d)].

\subsection{Measurement of the Permeability of the BBB}

In the general problem of substance transfer, two main contributions should be considered: a diffusive component and a convective one. Bulk convective flow in the brain arises because of communication between interstitial fluid and cerebrospinal fluid across the ependymal and pial surfaces. ${ }^{20}$ The very low permeability of the blood vessel walls means that there is negligible bulk flow into or out of the interstitium in other places. Because the extracellular spaces are narrow and filled with extracellular matrix, thermal convective flow can be neglected compared to diffusion.

The permeability coefficient, $P$, is defined by the equation ${ }^{7}$ :

$$
C_{e P}(t)=P \cdot \sigma \cdot \int_{0}^{t} C_{i P}(\tau) \mathrm{d} \tau,
$$

where $C_{e P}(t)$ is the extravascular concentration of the permeant molecule $P$ at time $t$, averaged over a region of interest (ROI), and $C_{i P}(\tau)$ is the plasma concentration at time $\tau$, with $\tau=0$ at the time of a bolus injection. $\sigma$ is the ratio of capillary surface area to extravascular volume in the ROI. The approxi- 
mation has been made that $C_{e P}(t) \ll C_{i P}(t)$. $C_{i P}(\tau)$ is obtained in parallel experiments by measurements in blood samples (see Sec. 3.2). Hence $P$ can be calculated if the ratio $C_{e P}(t) / C_{i P}(t)$ and $C_{i P}(0)$ are known.

The voxelized images of the dyes $\mathrm{P}$ and NP are described by functions $S_{P}(i, j, k)$ and $S_{N P}(i, j, k)$, where the coordinates $(i, j, k)$ specify the voxel in the image space that corresponds to the point $(x, y, z)$ in the tissue. The photomultiplier gains are adjusted so that the signals $S_{P}$ and $S_{N P}$ from the two dyes in the capillaries are equal:

$$
S_{i P}(i, j, k)=S_{i N P}(i, j, k) .
$$

$S_{P}$ and $S_{N P}$ are normalized so that the intensity of a pixel corresponding to a signal coming entirely from within a blood vessel is set to $2^{n}-1$ (Ref. 21), where $n$ is the number of bits of the analog to digital conversion. In the present experiment, $n=8$, giving a scale of 256 levels from 0 to 255 .

We found experimentally that the two signals could indeed be matched in all the capillaries. With the intravascular signals matched in this way, we define a difference signal:

$$
\operatorname{Diff}(i, j, k) \equiv S_{P}(i, j, k)-S_{N P}(i, j, k) .
$$

Since $S_{i P}(i, j, k)-S_{i N P}(i, j, k)=0, \operatorname{Diff}(i, j, k)$ is nonzero only in the extravascular space and is uncontaminated by signal from the intravascular dyes. It is shown in Sec. 7.3, Eq. (24), that the ratio

$$
\frac{C_{e P}(t)}{C_{i P}(t)} \equiv Q(t)=\frac{\sum \operatorname{Diff}(i, j, k, t)}{N},
$$

where $N$ is the number of pixels within the extravascular region of interest.

\subsection{Consequences of Bleed-Through Between the Detection Channels}

Equation (4) assumed that each detection channel was perfectly selective for the fluorescence from the corresponding dye. In practice, the separation was not perfect: as seen in Fig. 3 , the fluorescence spectrum from FITC-dextran extended into the bandpass of the SRB channel. However, it is simple to show algebraically that this bleed-through cancels when the NP image is subtracted from the P image [Sec. 7.4, Eqs. (25) and (26)]. If there were significant leakage of fluorescence from SRB into the FITC-dextran channel, it would affect the calculation, but inspection of Fig. 3 shows that, with the filter used, the leak is very small.

\section{Materials and Methods}

All animal experiments were performed in accordance with the French Government guidelines for the care and use of laboratory animals (License Nos. 380321, A 3851610004, and B 3851610003).

\subsection{Two-Photon Laser Scanning Microscopy}

The microscope consisted of an MRC 1024 scanhead (Biorad United Kingdom), and a BX50WI upright microscope (Olympus, Japan) fitted with a large, home-built, motorized stage (see schematic in Vérant et al. ${ }^{21}$ ). The optics could be

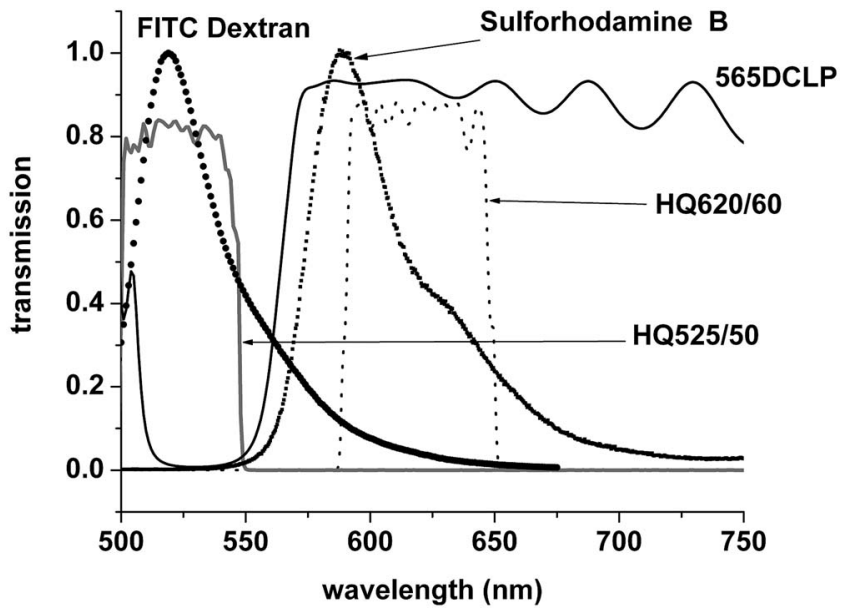

Fig. 3 Fluorescence spectra of FITC-dextran and SRB and the transmission curves of the interference filters used to select the fluorescence. 565DCLP is the transmission of the dichroic mirror.

switched between white light epi-illumination and observation and the multiphoton, laser scanning mode. An 800-nm excitation beam from a femtosecond Ti:Sapphire laser (Tsunami pumped by a Millennia V; Spectra-Physics, Inc., Mountain View, California) was focused into the sample using a $20 \times$ water-immersion objective, numerical aperture 0.95 , working distance of $2 \mathrm{~mm}$ (Xlum Plan FI, Olympus). The beam was scanned in the $x-y$ plane to acquire images 512 $\times 512$ pixels $(598 \mu \mathrm{m} \times 598 \mu \mathrm{m}, 0.9 \mathrm{~s} /$ image $)$. The observation depth was changed between images using the motor drive of the objective to obtain a Z-stack. The intensity of the incident laser beam was varied by rotating a half-wave plate in front of polarizers at Brewster's angle so that the total average power delivered at the surface ranged from 10 to $200 \mathrm{~mW}$. The confocal configuration was not used, the fluorescent light being diverted before reaching the scan head by a filter cube (HQ525-50, 565DCLP, HQ620-60, Chroma Technology Corp., Rockingham, Vermont). This directed the red fluorescence of SRB and the green fluorescence of FITCdextran separately onto two external photomultiplier tubes (Fig. 3). Images were displayed, as acquired, by the Biorad operating system.

\subsection{Fluorescent Probes}

For the dye that remains in the intravascular space, we chose $70 \mathrm{kDa}$ fluorescein isothiocyanate-dextran (FITC-dextran; Sigma-Aldrich, St. Quentin Fallavier, France), which does not cross the normal BBB in significant quantities in the times we consider $^{21}(<15 \mathrm{~min})$. We studied the extravasation of the much smaller (559 Da) sulforhodamine B (Lambda Physik, Göttingen, Germany), a polar, hydrophilic molecule. Neither of these is toxic for small animals at doses used in this work (see Material Safety Data Sheet ${ }^{22}$ ). Both dyes can be excited with two-photon transitions with femtosecond pulses of light centered at $800 \mathrm{~nm}$.

Both dyes are efficiently excited by two-photon absorption within the spectral range of the Ti:Sa laser, as shown on the $\mathrm{Xu}$ et al. ${ }^{23}$ spectra. 


\subsection{Microbeam Irradiation}

Swiss Nude mice (Charles River, L'Arbresle, France) approximately 5 weeks old, 14 to $24 \mathrm{~g}$, were anesthetized with xylazine/ketamione $(0.1 \% / 1 \%$ in buffered saline, $10 \mu \mathrm{l}$ per $\mathrm{g}$ body weight) and irradiated on the biomedical beamline ID17 of the European Synchrotron Radiation Facility, Grenoble, as described in Serduc et al. ${ }^{17}$ Briefly, the upper part of the left cerebral hemisphere was irradiated in the anteroposterior direction by an array of 18, vertically orientated, parallel, 25$\mu \mathrm{m}$-wide, microplanar beams; the distance between the centers was $211 \mu \mathrm{m}$. The entrance dose was $1000 \mathrm{~Gy}$ in a field $4 \mathrm{~mm} \times 4 \mathrm{~mm}$.

\subsection{Animal Preparation for Microscopy}

About $48 \mathrm{~h}$ after irradiation, the mice were anesthetized with isofluorane ( $5 \%$ for induction and $1.5 \%$ for maintenance), in a mixture of $70 \% \mathrm{~N}_{2} \mathrm{O}, 30 \% \mathrm{O}_{2}$. A cranial window $3 \mathrm{~mm}$ in diameter was made over the left parietal cortex, leaving the dura intact. The exposed brain surface was protected by a gel of $1 \%$ agarose in $0.9 \%$ saline solution. One hundred $\mu \mathrm{l}$ of a solution of $100 \mathrm{mg} \mathrm{ml}^{-1}$ FITC-dextran in $0.9 \%$ saline was added to $50 \mu \mathrm{l}$ of $10 \mathrm{mg} \mathrm{ml}^{-1} \mathrm{SRB}$ and injected in the tail vein. The mouse was then placed on a stereotaxic frame modified to permit rotation about the longitudinal axis; the cortical surface, where the laser light entered, could then be made horizontal. Imaging started within $5 \mathrm{~min}$ of the dye injection.

\subsection{Image Acquisition}

To avoid the large blood vessels at the surface of the brain, imaging started at a depth from the surface of about $150 \mu \mathrm{m}$. The offsets of the two photomultiplier tubes were first adjusted to avoid any artifactual signal background. The power of the laser beam was set initially to about $5 \mathrm{~mW}$, and during continuous $x, y$ scanning, the gains of the two detection channels, monitored by the photomultiplier supply voltages, were increased so as to produce the highest fluorescence signals possible without saturation in the images of the large blood vessels. The BioRad software indicates saturation of the digital signal in a voxel by a striking change of the false color, so this electronic matching of the images was readily performed. Planar scans of the fluorescent signals $S(x, y, z)$ were then acquired at successive depths in the cortex with a $z$-step between scans of 2 or $1.5 \mu \mathrm{m}$. As the depth increased, absorption and scattering of the exciting light and the emitted fluorescence reduced the image intensity. To compensate for this, the laser beam attenuation was reduced between scans, by manual control of the attenuator command, so as to keep the images of the large blood vessels close to saturated. The maximum power was about $200 \mathrm{~mW}$ (unfocused at the brain surface). A stack $200 \mu \mathrm{m}$ thick of 51 slices was acquired in $90 \mathrm{~s}$.

\subsection{Image Processing to Calculate $Q$}

Most of the processing algorithms are available on ImageJ (http://rsb.info.nih.gov/ij/).

1. Each image slice (a planar scan of $512 \times 512$ pixels corresponding to $598 \times 598 \mu \mathrm{m}^{2}$ ) of both the SRB and the FITC stacks ( $n$ slices) was convolved with a Gaussian function with a full width half maximum (FWHM) of 2 pixels corresponding to $2.3 \mu \mathrm{m}$. This step was necessary to eliminate isolated saturated pixels due to artifacts. Otherwise, such isolated intense pixels could have led to the normalization of step 2 failing to attribute the maximum intensity to large plunging vessels.

2. The pixel intensities for each slice were normalized on a scale from 0 to 1 (in 255 steps for an 8-bit acquisition) using the "enhance contrast" command of ImageJ. The maximum is the signal entirely from within a large vessel. ${ }^{21}$ This normalization automatically matches the intravascular signals $S_{i P}(i, j, k)$ and $S_{i N P}(i, j, k)$.

3. $\operatorname{Diff}(i, j, k) \equiv S_{P}(i, j, k)-S_{N P}(i, j, k) \quad[$ Sec. 7.2, Eq. (14)] was calculated for each pixel using homemade software written in Delphi-8 (Borland Software Corp., Cupertino, California).

4. The parameter $Q$ was then calculated from Eq. (4):

$$
Q=\frac{\sum \operatorname{Diff}}{N}=\frac{\sum_{i, j, k} \operatorname{Diff}(i, j, k)}{512 \times 512 \times n},
$$

for the case of $n$ slices, each $512 \times 512$ pixels. Note that this ratio is for the average intravascular and extravascular concentrations, the intravascular concentration being for whole blood, not plasma.

\subsection{Dye Clearance}

Equation (1), which defines the permeability coefficient $P$, requires knowledge of the concentration of SRB in the plasma $C_{i P}(t)$ at all times from the time of injection to the time of image acquisition. $C_{i P}(t)$ has been measured by Vérant et al. ${ }^{19}$ Briefly, a catheter was introduced in the jugular vein, and $100 \mu \mathrm{l}$ of an SRB solution $\left(5 \mathrm{mg} \mathrm{ml}^{-1}\right)$ was injected. (This is the same quantity as for the imaging experiments.) At different times up to $3 \mathrm{~h}$ after the injection, $40-\mu \mathrm{l}$ samples of blood were taken. The blood samples were diluted 41 times with heparinized physiological serum. One $\mathrm{ml}$ of each sample was centrifuged, and absorbance of the supernatant was measured at $560 \mathrm{~nm}$ with a spectrophotometer; the reference was made on heparinized physiological serum. The concentration of $\mathrm{SRB}$ in the plasma of the blood sample taken at time $t$ was calculated as:

$$
C_{i P}(t)=C_{\text {spect }} \times(41-H),
$$

where $C_{\text {spect }}$ is the concentration measured in the supernatant of the spectrometer sample and $H(\cong 0.42$; Ref. 24) is the hematocrit in the jugular vein.

\section{Results}

\subsection{Subtraction Imaging of Extravasated Dye}

Figures 2(a) and 2(b) show z-projected stacks of simultaneously acquired images of SRB and FITC-dextran fluorescence. The "maximum intensity" mode of ImageJ was used: this means that for any pixel of coordinate $\left(i_{m}, j_{n}\right)$ in the final image, only the most intense pixel $\left(i_{m}, j_{n}\right)$ in the z-stack is displayed. This mode shows the three-dimensional (3-D) organization of the object. The electronic gains of the two channels had been adjusted repeatedly during the acquisition of the stack so that the intensities of the blood vessels were the 

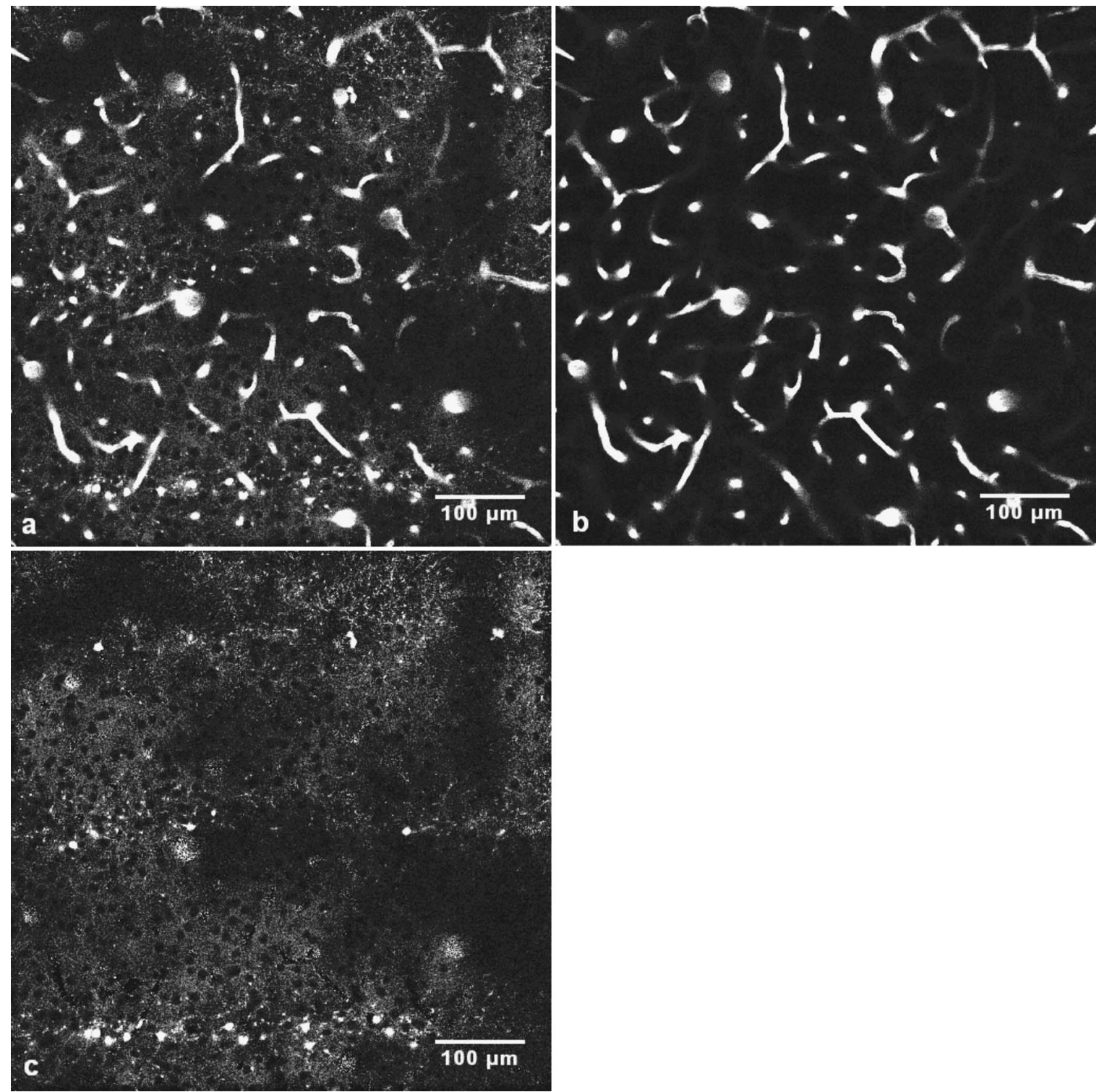

Fig. 4 Images obtained from the stacks of Fig. 2. (a) A slice image at a depth of $z=150 \mu \mathrm{m}$ below the dura for the SRB channel. (b) Same conditions, but for the FITC-dextran channel. (c) Image obtained by subtracting the FITC-dextran image from the SRB image.

same. The images of the blood vessels are almost identical, but in the SRB image, additional extravascular features can be made out in the form of three spotted stripes crossing the image horizontally. Each FITC-dextran slice image of the stack was then subtracted from the corresponding SRB slice image, and the resulting stack is displayed in two ways: in the "maximum intensity" mode [Fig. 2(d)], and in the "average intensity" mode [Fig. 2(c)]. In the "average intensity" mode, the intensities of each pixel $\left(i_{m}, j_{n}\right)$ in all slices were averaged to give the intensity in the final image. With either mode of display, the subtraction imaging almost completely eliminated the blood vessels and allowed the stripes to be seen more clearly [Figure 2(c) and 2(d)]. Such stripes were never seen in nonirradiated mice. The plane of the irradiation microbeams was vertical (in the $x, z$ plane of the stack), so their narrow profiles are seen in the image. The width (about $30 \mu \mathrm{m}$ ) and separation (about $200 \mu \mathrm{m}$ ) of the stripes clearly correspond to the geometry of the microbeams. ${ }^{17}$ The average diameter of the bright objects was $7.7 \mu \mathrm{m}, \mathrm{SD}=0.4 \mu \mathrm{m}$. We have not carried out experiments to identify these objects (see Sec. 5). In subtraction images of pairs of single optical slices, it was seen that, between stripes, there weredark objects, diameter about $10 \mu \mathrm{m}$, from which SRB was excluded [Fig. 4(c)]. The presence of these dark objects shows that the extravascular signal was not an artifact (due to an incorrectly set zero level, for example) and that SRB was indeed present in the extravascular tissue outside the bright $7.7 \mu \mathrm{m}$ objects. Figure 4(c) also shows that much of the extravascular SRB was concentrated in small granules, about $2 \mu \mathrm{m}$ in diameter.

\subsection{Measurement of the Ratio of Mean Extravascular to Intravascular Concentrations}

This is the first step toward determining a permeability coefficient, $P$, for $\mathrm{SRB}$ crossing the $\mathrm{BBB}$. We are interested in the 
total quantity of SRB in the extravascular space of the region of interest: the concentration of SRB in bright spots, and its exclusion from certain other volumes, will not change the estimation of $P$ (provided all the extravascular dye molecules fluorescence with equal efficiency). In Fig. 2(c), a z-projection of 76 slices of the subtracted images, we find that the ratio $Q$ of the mean extravascular to the intravascular concentration for the complete volume is [from Eq. (4)]:

$$
Q=\frac{\sum_{i, j, k} \operatorname{Diff}(i, j, k)}{512 \times 512 \times 76}=0.019,
$$

at time $t=10 \mathrm{~min}$ after the bolus infusion of dye.

When the stripes are excluded, for example, the rectangular ROI shown in Fig. 2(c) is $Q=0.017$, which is only $10 \%$ less than the value for the whole volume.

\subsection{Dye Clearance}

We write $C_{i P}(t)=C_{i P}(0) \times f(t)$, where $C_{i P}(0)$ is the plasma concentration immediately after the dye injection. It was found experimentally that:

$$
f(t)=0.68 \exp \left(-t / \tau_{1}\right)+0.27 \exp \left(-t / \tau_{2}\right)+0.045,
$$

where $\tau_{1}=0.36 \pm 0.4 \mathrm{~min}$ and $\tau_{2}=30 \pm 7 \mathrm{~min}$ (Vérant et al. $\left.{ }^{19}\right)$ The value of $C_{i P}(0)$, which is not necessary for the calculation of $P$, was $0.35 \mathrm{~g} / 1(0.63 \mathrm{mM})$.

\subsection{Calculation of Permeability}

$P$ is calculated from the dye concentration in the plasma, not in the whole blood. ${ }^{7}$ Therefore, $C_{e P}(t) / C_{i P}(t)$ (plasma) $=Q(t) /(1-H)$, where $H$ is the hematocrit in the mouse. Taking $H$ as 0.42 (Ref. 24) and $Q(t)=0.019$ gives $C_{e P}(t) / C_{i P}(t)=0.033$, for $t=10 \mathrm{~min}$. Using Eqs. (1) and (8), we calculate $P \cdot \sigma=1.7 \times 10^{-3} \mathrm{~min}^{-1} \quad$ which is $1.7 \mu \mathrm{l}$ (min. g of brain $)^{-1}$.

The specific capillary surface is given by $\sigma$ $=(C B V) \cdot 2 /\langle r\rangle$, where $\langle r\rangle$ is the mean capillary radius, and $C B V$ is the cerebral blood volume of the capillaries in the cortex. $C B V$ in the mouse is 0.022 (Ref. 21), and $\langle r\rangle$ measured from Fig. 2(b) is $2.4 \mu \mathrm{m}$. With these values, $\sigma=183 \mathrm{~cm}^{-1}$, and $P=10^{-6} \mathrm{~cm} / \mathrm{s}$. Note that this is a mean value for all the capillaries within the field of view.

\section{Discussion}

\subsection{Subtraction Imaging of Extravascular Space}

Injection of dye into the blood, with the aim of labeling structures within the parenchyma, has the advantage of being technically relatively simple and of introducing dye throughout the brain. This approach has previously been used to label amyloid plaques, ${ }^{25}$ and it is the only way of using dye to measure the permeability of the BBB. An obstacle has been that the weak extravascular fluorescence is difficult to measure in the presence of the intense fluorescence from the blood vessels. The subtraction method we have described here overcomes this difficulty.

As with sulforhodamine 101 applied to the cortex, ${ }^{26}$ we found that sulforhodamine B labeled specific objects [Fig. $2(d)]$. In our case, the labeled objects were in the tracks of the $\mathrm{x}$-ray microbeams, approximately $48 \mathrm{~h}$ after the irradiation. At this time, in brain sections stained with hematoxylin-eosin, nuclear condensation (pyknosis) is observed, and five days later, almost all cell bodies have disappeared from the microbeam tracks. ${ }^{17}$ Sulforhodamine B stains fixed cells in vitro. ${ }^{27}$ It is therefore possible that the stained objects were dying cells.

In tissues other than the brain, intravascular dyes pass readily into the tissue, ${ }^{28}$ which is presumably why the SRB was cleared so rapidly from the blood in the present experiments. This property has previously been used to introduce specific fluorescent markers into the kidney. ${ }^{29}$ In a brain with a normal BBB, entry of dyes into the parenchyma is much slower, but even here, there is some possibility of labeling from the blood.

\subsection{Measurement of BBB Permeability}

Our measurements gave a mean value of $P$ in the ROI of $10^{-6} \mathrm{~cm} / \mathrm{s}$. This is larger than the value of $0.5 \times 10^{-6} \mathrm{~cm} / \mathrm{s}$ for SRB obtained for pial venular capillaries in normal (unirradiated) rat brain by the single vessel occlusion technique ${ }^{5}$ but close to the value of $2 \times 10^{-6} \mathrm{~cm} / \mathrm{s}$ obtained under acute bradykinin treatment. ${ }^{5}$ As shown by Nicholson, ${ }^{30}$ a molecule the size of SRB will diffuse about $1000 \mu \mathrm{m}$ through brain interstitium in $10 \mathrm{~min}$, so with the present protocol, there was ample time for SRB to diffuse throughout the spaces between the irradiation tracks. Hence, the present results do not distinguish between a uniform increase in $P$ throughout the field of view and a localized increase only (or mainly) within the microbeam irradiation tracks. In the latter case (a local permeability increase), the local value of $P$ would be greater than the mean $P$ by the fraction (total volume/irradiated volume). This local $P$ would be $10^{-5} \mathrm{~cm} / \mathrm{s}$. Dialysis experiments have shown that part of the SRB in the blood is bound to albumin, so the values of $P$ for free SRB are underestimated ${ }^{19}$ by a factor (total concentration/free concentration) of 1.5.

These permeability values should be considered as crude because the hematocrit in small vessels is not exactly known, but certainly smaller than the value used here. In addition, the force that drives the diffusion is the concentration gradient across the vessel wall-all in fluid. The dye is measured in the tissue as a whole, which is approximately 5 times the volume of the ECS alone. These two considerations work in opposite directions, but we can estimate that our calculated permeability is underestimated by another factor of 2

\subsection{Advantages and Limitations of the Optical Subtraction Method for Measuring BBB Permeability}

Our preliminary measurement of the permeability of the BBB within the brain, at some depth from the surface, appears to be the first such measurement to use an optical technique in vivo. Unlike post mortem analysis of the distribution in sections of brain of a marked molecule (such as ${ }^{14} \mathrm{C}$ sucrose), the technique opens the possibility of making repeated measurements in one animal. In principle, it should be possible to follow a rapid change in $P$, over a few minutes, such as that induced by bradykinin, ${ }^{5}$ or to make repeated measurements at intervals of days or weeks, and follow slow changes, as has been done 
using two-photon microscopy to follow changes in neuronal morphology. 31

If the mean value of $P$ for the blood vessels within the region of interest (ROI) were greater than the mean value of $P$ outside the ROI, then there would be a net diffusional flux of dye out of the ROI, and this would lead to underestimation of $P$. The method therefore requires that any heterogeneity of $P$ extends throughout a region several times larger than the ROI. In contrast, this increase need not be uniform over distances small compared to the ROI. Alternatively, a highly localized increase in $P$ confined to a few $\mu \mathrm{m}^{2}$ of capillary endothelium might be studied by measuring $c_{e P}$ before significant quantities of the dye have diffused out of the ROI.

\subsection{Uneven Concentration of Extravasated Dye}

SRB in the extravascular space was not uniformly distributed, being concentrated in small granules, excluded from other objects, and within the irradiation tracks, concentrated in objects $7.7 \mu \mathrm{m}$ in diameter [Fig. 4(c)]. Provided the fluorescence efficiency (two-photon absorption cross section $\times$ fluorescence quantum efficiency) of the dye molecules remains the same as in the blood, this introduces no error in the measurement of $P$. SRB has been reported to bind to proteins in nonviable cells, ${ }^{27}$ and such binding may occur in the heavily stained objects in the microbeam tracks. However, it has been shown that binding of the SRB to proteins does not change its fluorescence. ${ }^{27}$

\section{Conclusion}

Injection of a nonpermeant dye together with one that crosses the BBB opens new possibilities both for imaging intraparenchymal features and for quantitative measurements of $\mathrm{BBB}$ permeability in vivo.

\section{Supplementary Material: Theory of Quantitative Subtraction Imaging}

We consider one dye that crosses the BBB, which we denote $\mathrm{P}$ (for permeant; in our case, SRB), and one dye that remains intravascular and is denoted NP (in our case, FITC-dextran).

\subsection{List of Symbols}

- $P$ (subscript) denotes dye that can cross the BBB. $P$ (capital) is the permeability coefficient of the BBB.

- $N P$ denotes dye that remains intravascular.

- $F_{i}(x, y, z)$ is a structure function describing the vascular space. $F_{i}(x, y, z)=1$ in the vascular space, 0 elsewhere.

- $F_{e}(x, y, z)$ describes the extravascular space. $F_{e}(x, y, z)$ $=0$ in the vascular space, 1 elsewhere.

- $\phi_{i}(x, y, z), \phi_{e}(x, y, z)$ denote convolutions of the structure functions with the point spread function, $\operatorname{PSF}(x, y, z)$. It is not necessary to know the PSF.

- $C_{i P}(x, y, z, t)$ is the intravascular concentration of dye P.

- $C_{i N P}(x, y, z, t)$ is the intravascular concentration of dye NP.

- $C_{e P}(x, y, z, t)$ is the extravascular concentration of dye $\mathrm{P}$. By definition, for the nonpermeant dye, $C_{e N P}(x, y, z, t)=0$.

- $E_{P}(x, y, z, t), E_{N P}(x, y, z, t)$ denote intensities of light emission of $\mathrm{P}$ and NP from point $(x, y, z)$ in the tissue.
- $S_{P}\left(x^{\prime}, y^{\prime}, z^{\prime}, t\right), S_{N P}\left(x^{\prime}, y^{\prime}, z^{\prime}, t\right)$ denote corresponding signals from point $\left(x^{\prime}, y^{\prime}, z^{\prime}\right)$ in the image space.

- $\alpha$ is a factor that accounts for bleed-through of light from the shorter wavelength dye (FITC-dextran) into the longer wavelength channel.

- $\beta$ is the corresponding factor that accounts for bleedthrough of light from the longer wavelength dye (SRB) into the shorter wavelength channel.

- $Q(R, t)$ denotes the ratio of extravascular to intravascular concentrations of the permeant dye $\mathrm{P}$ in the region of interest $R$.

- $V_{i} / V_{e}$ is the specific blood volume. (Strictly, it is $V_{i} /\left(V_{e}+V_{i}\right)$, but $\left.V_{e} \gg V_{i}\right)$

\subsection{Subtraction Imaging}

The image of a fluorescent capillary extends, with a low intensity, well beyond the geometrical limits of the capillary [Fig. 1(c)]. Hence, a significant part of the acquired signal attributed to a point in the extravascular space comes, in practice, not from extravasated dye but from the much more intense fluorescence of the more concentrated dye within the capillaries [difference between solid and dashed lines in Fig. 1(b)]. This problem is overcome by using a second dye that remains intravascular.

In two-photon microscopy, the fraction of the incident light is small so that the fluorescence is proportional to the concentration of the dye, $C$, and also to its absorption coefficient, to the quantum efficiency of the fluorescence, and to the incident intensity of the exciting light. The latter factors are combined in a constant $K$ for each dye. Hence, the intensity of emitted light for the permeant dye $P$ is given by:

$$
\begin{aligned}
E_{P}(x, y, z, t)= & K_{P} C_{e P}(x, y, z, t) F_{e}(x, y, z) \\
& +K_{P} C_{i P}(x, y, z, t) F_{i}(x, y, z),
\end{aligned}
$$

where the structure functions, $F_{e}$ and $F_{i}$, which can have values of 0 or 1 , describe the extravascular and intravascular spaces.

By definition, for the nonpermeant dye, $C_{e N P}=0$ and

$$
E_{N P}(x, y, z, t)=K_{N P} C_{i N P}(x, y, z, t) F_{i}(x, y, z) .
$$

The distribution of the intensities of the pixels in the acquired image differs from $E(x, y, z)$. The main reason is that the laser beam is not focused to a perfect point but excites fluorophores in a volume that can be described by a point spread function (see Vérant et al. ${ }^{21}$ ). For quantitative measurements of the extravascular concentration, $C_{e P}(x, y, z, t)$, it is necessary that the detection chain be linear (see below), but for qualitative detection of weak extravascular fluorescence, it is permissible to increase the gains of the two channels until the relatively intense fluorescence from the blood vessels starts to saturate the amplifiers. We denote the combined optical and electronic effects by a function $\operatorname{PSF}(x, y, z)$, which is assumed to be the same for both $\mathrm{P}$ and NP. We then show that the subtraction method makes it unnecessary to know $\operatorname{PSF}(x, y, z)$. In the images, the structure functions $F$ are replaced by their convolutions $\phi_{i}(x, y, z)$ with the $P S F$ 


$$
\begin{aligned}
\phi(x, y, z)= & \int_{-\infty}^{\infty} \int_{-\infty}^{\infty} \int_{-\infty}^{\infty} F(\xi, \psi, \zeta) \operatorname{PS} F(x-\xi, y-\psi, z \\
& -\zeta) \mathrm{d} \xi \mathrm{d} \psi \mathrm{d} \zeta .
\end{aligned}
$$

We define the images by pixel intensities $S(t)$ in an image space $\left(x^{\prime}, y^{\prime}, z^{\prime}, t\right)$ :

$$
S_{p}\left(x^{\prime}, y^{\prime}, z^{\prime}, t\right) \Leftrightarrow k_{p} C_{e P}(x, y, z, t) \phi_{e}(x, y, z)+k_{p} C_{i P}(t) \phi_{i}(x, y, z),
$$

$$
S_{N P}\left(x^{\prime}, y^{\prime}, z^{\prime}, t\right)=S_{i N P}\left(x^{\prime}, y^{\prime}, z^{\prime}, t\right) \Leftrightarrow k_{N P} C_{i N P}(t) \phi_{i}(x, y, z),
$$

where $k_{P}$ and $k_{N P}$ are new constants that include $K_{P}$ and $K_{N P}$ and also take into account the properties of the detection chain.

These expressions suppose a moderate spatial variation of the extravascular dye concentration compared with the scale of the PSF.

We now adjust the gains of the two detection chains so that the signals from the vascular space are equal:

$$
S_{i P}\left(x^{\prime}, y^{\prime}, z^{\prime}, t\right)=S_{i N P}\left(x^{\prime}, y^{\prime}, z^{\prime}, t\right)(\text { intravascular space }) .
$$

We found experimentally that the two signals could indeed be matched throughout the vascular space, as would be expected provided the two dyes are both excluded from the blood cells, as is the case for SRB and FITC-dextran. From Eqs. (11) and (12):

$$
k_{P} C_{i P}(x, y, z, t)=k_{N P} C_{i N P}(x, y, z, t) .
$$

We define a difference signal $\operatorname{Diff}\left(x^{\prime}, y^{\prime}, z^{\prime}, t\right)$ obtained by subtracting Eq. (11b) from Eq. (11a):

$$
\begin{aligned}
\operatorname{Diff}\left(x^{\prime}, y^{\prime}, z^{\prime}, t\right) \equiv & S_{p}\left(x^{\prime}, y^{\prime}, z^{\prime}, t\right)-S_{N P}\left(x^{\prime}, y^{\prime}, z^{\prime}, t\right) \\
& \Leftrightarrow k_{p} C_{e P}(x, y, z, t) \phi_{e}(x, y, z) .
\end{aligned}
$$

Hence, within the resolution of the two-photon microscopy, $\operatorname{Diff}\left(x^{\prime}, y^{\prime}, z^{\prime}, t\right)$ is present only in the extravascular space and is uncontaminated by signals from the intravascular dyes. Provided the two intravascular signals are matched, the gain can be increased almost without limit to reveal extracellular features.

\subsection{Quantification of Dye Extravasation: Ratio of Extravascular Concentration to Intravascular Concentration}

Let $Q(R, t)$ be the ratio between extravascular and intravascular concentrations of the permeant dye $P$ in the region of interest $R$, at a given time $t$ :

$$
Q(R, t)=\frac{C_{e P}(R, t)}{C_{i P}(R, t)},
$$

where $C_{e P}(R, t)$ is the average concentration of $P$ in region $R$. When the intravascular signals are matched [Eqs. (12) and (13)]:

$$
\begin{gathered}
C_{i P}(R, t)=C_{i N P}(R, t) k_{N P} / k_{P}, \\
Q(R, t)=\frac{C_{e P}(R, t)}{C_{i N P}(R, t)} \frac{k_{P}}{k_{N P}} .
\end{gathered}
$$

From Eq. (11b), summating over the pixels in region $R$, at time $t$ :

$$
k_{N P} \cdot C_{I N P}=\frac{\sum S_{N P}}{\sum \phi_{i}} .
$$

Provided that $R$ covers a large number of pixels, the sum of the convolution of the intravascular function equals the sum of the intravascular function itself:

$$
\sum \phi_{i}=\sum F_{i}
$$

So Eq. (18) becomes:

$$
k_{N P} \cdot C_{i N P}=\frac{\sum S_{N P}}{\sum F_{i}},
$$

and similarly Eq. (14) gives:

$$
k_{P} \cdot C_{e P}=\frac{\sum D i f f}{\sum F_{e}} .
$$

In Eqs. (20) and (21), the summations over $F_{i}$ and $F_{e}$ give the intravascular volume $V_{i}$ and the extravascular volume $V_{e}$ within the region of interest. So the final expression for $Q$ becomes:

$$
Q=\frac{\sum \text { Diff }}{\sum S_{N P}} \frac{V_{i}}{V_{e}} .
$$

The ratio $V_{i} / V_{e}$ is the specific blood volume, which as shown by Vérant et al. ${ }^{21}$ can also be calculated by summing over the same region of interest:

$$
\frac{V_{i}}{V_{e}}=\frac{\sum S_{N P}}{N},
$$

where $S_{N P}$ has been scaled (normalized) in each image slice of the stack to be unity in pixels corresponding to fluorescence that is entirely intravascular, and $N$ is the total number of pixels within the region of interest.

We finally obtain:

$$
Q=\frac{\sum \text { Diff }}{N},
$$

where the scale of Diff follows from the normalizations of $S_{N P}$ and $S$, as explained in Sec. 2.2. 


\subsection{Consequences of Bleed-Through Between the two Detection Channels}

Eq. (9) assumed that each photomultiplier detected light emitted by only one dye. As seen in Fig. 3, the fluorescence spectrum from FITC-dextran extends into the bandpass of the SRB channel and makes a contribution that we denote as fraction $\alpha$ of its detection in the FITC-dextran channel, and conversely, a very small amount of fluorescence, $\beta$, from SRB extends into the FITC-dextran bandpass. Adding terms to Eq. (11) to account for this gives:

$$
\begin{gathered}
S_{P}=k_{P} C_{i P} \Phi i+\alpha k_{N P} C_{i N P} \Phi i+k_{P} C_{e P} \Phi e \\
S_{N P}=k_{N P} C_{i N P} \Phi i+\beta k_{P} C_{i P} \Phi i+\beta k_{P} C_{e P} \Phi e .
\end{gathered}
$$

The signals from intravascular space are adjusted to match:

$$
k_{P} C_{i P} \Phi i+\alpha k_{N P} C_{i N P} \Phi i=k_{N P} C_{i N P} \Phi i+\beta k_{P} C_{i P} \Phi I .
$$

Hence:

$$
\operatorname{Diff}(x y z) \equiv S_{P}-S_{N P}=(1-\beta) k_{P} C_{e P} \Phi e .
$$

We see that the $\alpha$ term is eliminated, leaving only $\beta$, which by inspection of Fig. 3, is very small.

\subsection{Measurement of the Permeability of the $B B B$}

The transport across the $\mathrm{BBB}$ of a substance that is not carried by transporter molecules follows from Fick's Law ${ }^{7}$ :

$$
\frac{\mathrm{d} C_{e P}(t)}{\mathrm{d} t}=\frac{P \cdot S}{V_{e}} \cdot\left[C_{i P}(t)-C_{e P}^{\prime}(t)\right],
$$

where $S$ is the surface area of the blood vessels in the region of interest, $V_{e}$ is the extravascular volume, the equation defines a permeability constant $P$, and $C_{e P}^{\prime}(t)$ is the concentration immediately outside the BBB. However, molecules of MWt up to about 10,000 diffuse freely through the extracellular clefts of brain tissue, and $\mathrm{Wu}$ et al. ${ }^{12}$ have estimated that provided the measurement is made at a time sufficiently short that mean $C_{e P}(t) \ll C_{i P}(t)$, then $C_{e P}^{\prime}(t) \ll C_{i P}(t)$. Hence:

$$
\frac{\mathrm{d} C_{e P}(t)}{\mathrm{d} t} \cong \sigma P \cdot C_{i P}(t)
$$

where $\sigma=S / V_{e}$. We write:

$$
C_{i P}(t) \equiv C_{i P}(0) \cdot f(t)
$$

where $C_{i P}(0)$ is the intravascular concentration just after the injection, at $t=0$, and $f(t)$ is the time-course of the decline in $C_{i P}$ as the dye is cleared from the blood. Integration gives:

$$
C_{e P}(t)=P \cdot \sigma \cdot C_{i P}(0) \cdot \int_{0}^{t} f(\tau) \mathrm{d} \tau .
$$

From Eqs. (15) and (29),

$$
C_{e P}(t)=Q(t) \cdot C_{i P}(0) \cdot f(t)
$$

Hence:

$$
P=Q(t) \cdot \frac{f(t)}{\sigma \int_{0}^{t} f(t) \mathrm{d} t} .
$$

Inserting Eq. (24) for $Q(t)$ :

$$
P=\frac{\sum \operatorname{Diff}(t)}{N} \cdot \frac{f(t)}{\sigma \int_{0}^{t} f(t) \mathrm{d} t} .
$$

$f(t)$ is obtained from measurements of $C_{P}$ in the blood.

\section{Acknowledgments}

This work had been supported by grants from Ligue Contre le Cancer (Comité de l'Isère), Association pour la Recherche sur le Cancer, Programme Interdisciplinaire CNRS-INSERMCEA «IPA», and Région Rhône-Alpes (Appel d'Offre Thématique Cancer). Raphaël Serduc received a grant from La Ligue Contre le Cancer.

\section{References}

1. I. Klatzo, "Pathophysiological aspects of brain edema," Acta Neuropathol. (Berl) 72, 236-239 (1987).

2. D. M. Hermann, E. Kilic, A. Spudich, S. D. Kramer, H. WunderliAllenspach, and C. L. Bassetti, "Role of drug efflux carriers in the healthy and diseased brain," Ann. Neurol. 60, 489-498 (2006).

3. W. M. Pardridge, "Blood-brain barrier biology and methodology," $J$. Neurovirol 5, 556-569 (1999).

4. E. Oby and D. Janigro, "The blood-brain barrier and epilepsy," Epilepsia 47, 1761-1774 (2006).

5. M. H. Sarker, D. E. Hu, and P. A. Fraser, "Acute effects of bradykinin on cerebral microvascular permeability in the anaesthetized rat," J. Physiol. (London) 528(1), 177-187 (2000).

6. M. A. Petty and E. H. Lo, "Junctional complexes of the blood-brain barrier: permeability changes in neuroinflammation," Prog. Neurobiol. 68, 311-323 (2002).

7. C. S. Patlak, R. G. Blasberg, and J. D. Fenstermacher, "Graphical evaluation of blood-to-brain transfer constants from multiple-time uptake data," J. Cereb. Blood Flow Metab. 3, 1-7 (1983).

8. B. T. Hawkins and R. D. Egleton, "Fluorescence imaging of bloodbrain barrier disruption," J. Neurosci. Methods 151, 262-267 (2006).

9. R. Sood, S. Taheri, E. Y. Estrada, and G. A. Rosenberg, "Quantitative evaluation of the effect of propylene glycol on BBB permeability," $J$. Magn. Reson Imaging 25, 39-47 (2007).

10. S. Taheri and R. Sood, "Kalman filtering for reliable estimation of BBB permeability,” J. Magn. Reson Imaging 24, 1039-1049 (2006).

11. S. Syvanen, G. Blomquist, L. Appel, M. Hammarlund-Udenaes, B. Langstrom, and M. Bergstrom, "Predicting brain concentrations of drug using positron emission tomography and venous input: modeling of arterial-venous concentration differences," Eur. J. Clin. Pharmacol. 62, 839-848 (2006).

12. N. Z. Wu, B. Klitzman, G. Rosner, D. Needham, and M. W. Dewhirst, "Measurement of material extravasation in microvascular networks using fluorescence video-microscopy," Microvasc. Res. 46, 231-253 (1993).

13. A. Bultmann, L. Schilling, A. Findling, and M. Wahl, "Investigation of blood-brain barrier permeability by means of computerized image analysis," Vasa 23, 195-204 (1994).

14. J. Takahashi, F. Mori, T. Hikichi, and A. Yoshida, "Effect of acetazolamide on outward permeability of blood-retina barrier using differential vitreous flyorophotometry," Curr. Eye Res. 23, 166-170 (2001).

15. W. Denk, J. H. Strickler, and W. W. Webb, "Two-photon laser scanning fluorescence microscopy," Science 248, 73-76 (1990).

16. D. Kleinfeld, P. P. Mitra, F. Helmchen, and W. Denk, "Fluctuations and stimulus-induced changes in blood flow observed in individua capillaries in layers 2 through 4 of rat neocortex," Proc. Natl. Acad. Sci. U.S.A. 95, 15741-15746 (1998). 
17. R. Serduc, P. Vérant, J. C. Vial, R. Farion, L. Rocas, C. Rémy, T Fadlallah, E. Brauer, A. Bravin, J. Laissue, H. Blattmann, and B van-der-Sanden, "In vivo two-photon microscopy study of short-term effects of microbeam irradiation on normal mouse brain microvasculature," Int. J. Radiat. Oncol., Biol., Phys. 64, 1519-1527 (2006).

18. J. A. Laissue, G. Geiser, P. O. Spanne, F. A. Dilmanian, J. O. Gebbers, M. Geiser, X. Y. Wu, M. S. Makar, P. L. Micca, M. M. Nawrocky, D. D. Joel, and D. N. Slatkin, "Neuropathology of ablation of rat gliosarcomas and contiguous brain tissues using a microplanar beam of synchrotron-wiggler-generated $\mathrm{x}$ rays," Int. J. Cancer 78, 654-660 (1998).

19. P. Vérant et al., to be published, and P. Vérant, Thesis in Physical Sciences, University Joseph Fourier, Grenoble, France (2006).

20. H. F. Cserr, M. Depasquale, C. S. Patlak, R. G. L. Pullen, "Convection of cerebral interstitial fluid and its role in brain volume regulation," Ann. N.Y. Acad. Sci. 481, 123-133. (1987)

21. P. Vérant, R. Serduc, B. van-der-Sanden, C. Remy, and J. C. Vial, "A direct method for measuring mouse capillary cortical blood volume using multiphoton laser scanning microscopy," J. Cereb. Blood Flow Metab. 27, 1072-1081 (2007).

22. http://www.sciencelab.com/msdsList.php.

23. C. Xu and W. W. Webb, "Measurement of two-photon excitation cross sections of molecular fluorophores with data from 690 to 1050 nm," J. Opt. Soc. Am. B 13, 481-491 (1996).

24. J. Vogel, I. Kiessling, K. Heinicke, T. Stallmach, P. Ossent, O. Vogel, M. Aulmann, T. Frietsch, H. Schmid-Schonbein, W. Kuschinsky, and
M. Gassmann, "Transgenic mice overexpressing erythropoietin adapt to excessive erythrocytosis by regulating blood viscosity," Blood $\mathbf{1 0 2}$, 2278-2284 (2003).

25. E. M. Robbins, R. A. Betensky, S. B. Domnitz, S. M. Purcell, M. Garcia Alloza, C. Greenberg, G. W. Rebeck, B. T. Hyman, S. M. Greenberg, M. P. Frosch, and B. J. Bacskai, "Kinetics of cerebral amyloid angiopathy progression in a transgenic mouse model of Alzheimer disease," J. Neurosci. 26, 365-371 (2006).

26. A. Nimmerjahn, F. Kirchhoff, J. N. Kerr, and F. Helmchen, "Sulforhodamine 101 as a specific marker of astroglia in the neocortex in vivo," Nat. Methods 1, 31-37 (2004).

27. P. Skehan, R. Storeng, D. Scudiero, A. Monks, J. McMahon, D. Vistica, J. T. Warren, H. Bokesch, S. Kenney, and M. R. Boyd, "New colorimetric cytotoxicity assay for anticancer-drug screening," $J$. Natl. Cancer Inst. 82, 1107-1112 (1990).

28. G. Bouffard, "Injection des couleurs de benzidine aux animaux normaux," Ann. Inst. Pasteur (Paris) 20, 539 (1906).

29. K. W. Dunn, R. M. Sandoval, K. J. Kelly, P. C. Dagher, G. A. Tanner, S. J. Atkinson, R. L. Bacallao, and B. A. Molitoris, "Functional studies of the kidney of living animals using multicolor two-photon microscopy," Am. J. Physiol.: Cell Physiol. 283, 905-916 (2002).

30. C. Nicholson, "Diffusion and related transport mechanisms in brain tissue," Reports on Progress in Physics 64, 815-884 (2001).

31. A. K. Majewska, J. R. Newton, and M. Sur, "Remodeling of synaptic structure in sensory cortical areas in vivo," J. Neurosci. 26, 30213029 (2006). 